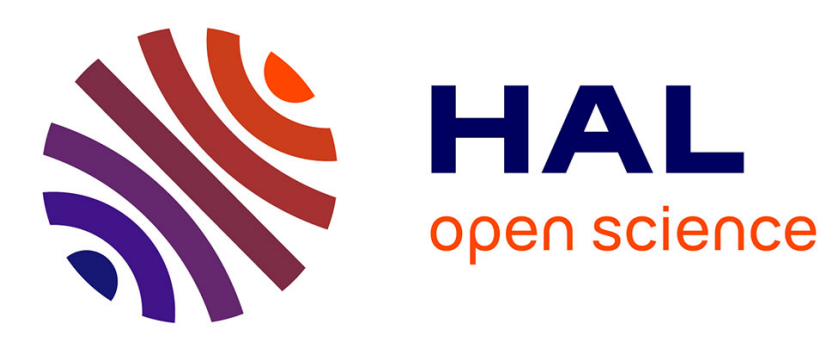

\title{
Approximate distribution of the low-rank adaptive normalized matched filter test statistic under the null hypothesis
}

Guillaume Ginolhac, Philippe Forster

\section{- To cite this version:}

Guillaume Ginolhac, Philippe Forster. Approximate distribution of the low-rank adaptive normalized matched filter test statistic under the null hypothesis. IEEE Transactions on Aerospace and Electronic Systems, 2016, 52 (4), pp.2016 - 2023. 10.1109/TAES.2016.150042 . hal-01503775

\section{HAL Id: hal-01503775 \\ https://hal.science/hal-01503775}

Submitted on 7 Apr 2017

HAL is a multi-disciplinary open access archive for the deposit and dissemination of scientific research documents, whether they are published or not. The documents may come from teaching and research institutions in France or abroad, or from public or private research centers.
L'archive ouverte pluridisciplinaire HAL, est destinée au dépôt et à la diffusion de documents scientifiques de niveau recherche, publiés ou non, émanant des établissements d'enseignement et de recherche français ou étrangers, des laboratoires publics ou privés. 


\title{
Approximate Distribution of the Low-Rank Adaptive Normalized Matched Filter Test Statistic Under the Null Hypothesis
}

\author{
Guillaume Ginolhac, Member, IEEE, Philippe Forster, Member, IEEE
}

\begin{abstract}
In this paper, we propose to derive an approximate theoretical distribution under the null hypothesis of the Low-Rank Adaptive Normalized Matched Filter (LR-ANMF). This detector is used to detect a target when the disturbance is composed of a Low-Rank Gaussian contribution (called clutter) and an Additive White Gaussian Noise (AWGN). In the LR-ANMF, the estimated covariance matrix is replaced by the estimated orthogonal projector onto the subspace clutter. The method to derive this distribution is based on a perturbation analysis and assumes that the steering vector is far from the clutter and a large Clutter-to-Noise Ratio (CNR). Simulations on a STAP example validate our theoretical result and the impact of both hypotheses is also studied.
\end{abstract}

\section{Index Terms}

Adaptive detection, Low-Rank, Approximate Distribution, Perturbation Analysis, STAP

\section{INTRODUCTION}

Several applications, e.g. RADAR or SONAR, consist of detecting a known signal, the so-called steering vector, embedded in a disturbance. In this context, Likelihood Ratio Test and Generalized Likelihood Ratio Test (GLRT) have been developed. When the covariance matrix of the disturbance is known, the theoretical performances of the developed detectors are well known even for subspace signals [1], [2]. But in practice the covariance matrix is unknown. From so-called secondary data, assumed to be independent and to share the same distribution as the observation under test, E.J. Kelly [3] derived the Generalized

Guillaume Ginolhac is with LISTIC - Université Savoie Mont Blanc, email: guillaume.ginolhac@univ-smb.fr. Philippe Forster is with SATIE - ENS Cachan, email: philippe.forster@satie.ens-cachan.fr. 
Likelihood Ratio Test (GLRT). The theoretical Pfa and Pd has been also computed. Another solution has been proposed by [4]: the detector is first derived by assuming a known covariance matrix and the Maximum Likelihood Estimator (MLE) of the covariance matrix is then plugged in the previous detector. The theoretical Pfa and Pd have been also computed and have showed close performance compared to Kelly's detector.

Nevertheless, these detectors need a large number of secondary data, $K$, to reach correct performance, i.e. $K \approx 2 m$ (where $m$ is the data size) [5], [6]. Since this number $m$ may be large in some applications (e.g. STAP), it is important to derive detection techniques for small $K$ compared to $m$. To achieve this goal, many ways have been and are still investigated in the array processing community. For example, a Reduced-Rank (RR) STAP algorithm based on an AR model is proposed in [7]. In [8], a combination between the possible persymetric structure of the covariance matrix and the Extented Factor Approach (EFA) allows a great reduction of the number $K$. Li and al propose in [9] a new cost function to build an EFA algorithm based on a low-rank approximation. Another approach is to integrate a priori information about the clutter to improve STAP filtering [10][11]. For slow moving target, [12] derives a RR STAP based on a min-max algorithm. When the disturbance is structured as a sum of an Additive White Gaussian Noise (AWGN) and a Low-Rank (LR) contribution (the so-called clutter in RADAR or SONAR) this number $K$ of secondary data can also be reduced. For example, the LR adaptive filtering only needs $K=2 r$ (where $r$ is the clutter rank) when classical adaptive filtering needs $K=2 m$ to reach the same performance [13], [14]. In a context of a disturbance composed of a LR clutter and an AWGN and by assuming to know the projector onto the subspace clutter, we obtain the LR Normalized Matched Filter (LR-NMF) [2][15][16]. Its theoretical performance are obtained in [15]. Since the covariance matrix of the clutter is not known in practice, it has to be estimated from secondary data. The structure information about the reduced rank of the clutter covariance matrix can be taken into account in the covariance estimation step in order to improve the detection performance. For example, several recent works propose to constraint the estimation of the covariance matrix to be reduced rank [17], [18], [19], [20], [21] in order to improve the accuracy of the estimation. In these works, the proposed algorithms estimate the eigenvectors and the eigenvalues (and other parameters in non gaussian case) of the covariance matrix. In this paper, we are only interested in the estimated eigenvectors to build the estimated projector onto the subspace clutter. In a gaussian clutter, it is well known that the Maximum Likelihood Estimator is then obtained through the Eigen-Value Decomposition (EVD) of the Sample Covariance Matrix (SCM). By plugging this estimate in the LR-NMF, we obtain the so-called LR Adaptive Normalized Matched Filter (LR-ANMF). Other LR adaptive detectors have also been developed and can be found in [22], 
[23], [24]. Unfortunately for all these detectors and in particular for the LR-ANMF, the theoretical Pfa and $\mathrm{Pd}$ are not derived in the literature.

We propose in this paper to derive an approximate theoretical distribution of the LR-ANMF test statistic under the null hypothesis by means of a perturbation analysis [25]. As in previous works [13], [14], [26], [27], [28] on theoretical performances based on this approach, we assume the steering vector is orthogonal with respect to the clutter subspace for mathematical tractability. We also assume that the Clutter-to-Noise Ratio (CNR) is large which is a common assumption in RADAR or SONAR. Numerical simulations are performed for a Space-Time Adaptive Processing (STAP) application. STAP is a technique used in airborne phased array radar to detect moving target embedded in an interference background such as jamming or strong clutter [29]. While conventional radars are able to detect target both in the time domain related to target range and in the frequency domain related to target velocity, STAP uses an additional domain (space) related to the target angular localization. The consequence is a two-dimensional adaptive filtering technique which uses jointly temporal and spatial dimensions to cancel interference and to improve target detection. In most cases, the disturbance in STAP is known to be composed of a LR-clutter plus an AWGN where the rank is easily deduced [30]. Results in this context validate our theoretical result even in the cases of non orthogonality of the steering vector with respect to the clutter subspace and of low CNR.

Paper is organized as follows: section II presents the problem statement, section III contains the main result of the paper which is the approximate distribution of the LR-ANMF under $H_{0}$ hypothesis and section IV shows the results of different numerical simulations.

The following convention is adopted: italic indicates a scalar quantity, lower case boldface indicates a vector quantity and upper case boldface a matrix. ${ }^{T}$ denotes the transpose operator and ${ }^{H}$ the transpose conjugate. $E[]$ is the expected value operator. $\mathcal{C N}(\mathbf{a}, \mathbf{M})$ is a complex Gaussian vector of mean a and of covariance matrix M. $\mathbf{I}_{m}$ is the $m \times m$-identity matrix. $\chi^{2}(n)$ is a Chi-square random variable with $n$ degrees of freedom. $\mathscr{N}_{c}\left(a, \sigma^{2}\right)$ is complex Gaussian random variable of mean $a$ and variance $\sigma^{2}$. Vector $\mathbf{e}_{i}$ is the vector with only one non-zero component equal to 1 at index $i$. $\sim$ means "distributed as".

\section{Problem Statement}

The stated problem is to infer if the received signal $\mathbf{x} \in \mathbb{C}^{m \times 1}$, corrupted by an additive disturbance, also contains a complex signal $\mathbf{a}$. One also has a set of $K$ secondary data $\left\{\mathbf{x}_{k}\right\}$ which are signal free 
realizations of the disturbance. The two hypotheses are then:

$$
\left\{\begin{array}{lll}
H_{0}: \mathbf{x}=\mathbf{c}+\mathbf{n} & \mathbf{x}_{k}=\mathbf{c}_{k}+\mathbf{n}_{k}, & k \in \llbracket 1, K \rrbracket \\
H_{1}: \mathbf{x}=\mathbf{a}+\mathbf{c}+\mathbf{n} & \mathbf{x}_{k}=\mathbf{c}_{k}+\mathbf{n}_{k}, & k \in \llbracket 1, K \rrbracket
\end{array}\right.
$$

$\mathbf{a}$ is the desired signal and is equal to $\alpha \mathbf{d}(\boldsymbol{\Theta})$ where $\mathbf{d}$ is the steering vector, $\alpha$ is an unknown deterministic parameter and $\Theta$ is an unknown deterministic vector. $\mathbf{n} \in \mathbb{C}^{m \times 1}$ (or $\left.\mathbf{n}_{k}\right) \sim \mathcal{C N}\left(0, \lambda \mathbf{I}_{m}\right)$ is the AWGN complex vector. $\mathbf{c} \in \mathbb{C}^{m \times 1}$ (or $\left.\mathbf{c}_{k}\right) \sim \mathcal{C N}(0, \mathbf{C})$ is the Gaussian clutter. Consequently, the covariance matrix of the secondary data can be written as $\mathbf{R}=\mathbf{C}+\lambda \mathbf{I}_{m} \in \mathbb{C}^{m \times m}$. Moreover, the clutter is considered of low-rank $r^{1}$. Hence, $\operatorname{rank}(\mathbf{C})=r \ll m$ and one could write the eigendecomposition of $\mathbf{C}$ and define:

$$
\mathbf{C}=\sum_{i=1}^{r} \lambda_{i} \mathbf{u}_{i} \mathbf{u}_{i}^{H}
$$

where $\lambda_{i}$ and $\mathbf{u}_{i}, i \in \llbracket 1 ; r \rrbracket$ are respectively the non zero eigenvalues and the associated eigenvectors of C.

We define the following unitary matrices:

$$
\begin{aligned}
\mathbf{U}_{c} & =\left[\begin{array}{llll}
\mathbf{u}_{1} & \mathbf{u}_{2} & \ldots & \mathbf{u}_{r}
\end{array}\right] \\
\mathbf{U}_{0} & =\left[\begin{array}{llll}
\mathbf{u}_{r+1} & \mathbf{u}_{r+2} & \ldots & \mathbf{u}_{m}
\end{array}\right]
\end{aligned}
$$

where $\mathbf{u}_{i}, i \in \llbracket r+1 ; m \rrbracket$ are the eigenvectors associated to the eigenvalue $\lambda$.

We define the projector onto the clutter subspace $\boldsymbol{\Pi}_{c}$ and the projector onto the orthogonal of the clutter subspace $\boldsymbol{\Pi}_{c}^{\perp}=\mathbf{I}_{m}-\boldsymbol{\Pi}_{c}[13],[14]$ :

$$
\begin{aligned}
\boldsymbol{\Pi}_{c} & =\sum_{i=1}^{r} \mathbf{u}_{i} \mathbf{u}_{i}^{H} \\
\boldsymbol{\Pi}_{c}^{\perp} & =\mathbf{I}_{m}-\sum_{i=1}^{r} \mathbf{u}_{i} \mathbf{u}_{i}^{H}
\end{aligned}
$$

A preprocessing on the observation vector is first done in order to remove the clutter, and we retrieve a complex signal detection problem defined by the following binary hypothesis test:

$$
\left\{\begin{array}{lll}
H_{0}: \mathbf{r}=\mathbf{U}_{0}^{H} \mathbf{x}=\mathbf{n}_{0} & \mathbf{r}_{k}=\mathbf{n}_{0, k}, & k \in \llbracket 1, K \rrbracket \\
H_{1}: \mathbf{r}=\mathbf{U}_{0}^{H} \mathbf{x}=\mathbf{d}_{0}+\mathbf{n}_{0} & \mathbf{r}_{k}=\mathbf{n}_{0, k}, & k \in \llbracket 1, K \rrbracket
\end{array}\right.
$$

The detection problem is solved considering the white noise power $\mathbf{n}_{0}$ unknown. The used detection test corresponds to the Normalized Matched Filter in its low-rank version, denoted by LR-NMF [15]:

$$
\Lambda_{\mathrm{LR}}(\boldsymbol{\Theta})=\frac{\left|\mathbf{d}(\boldsymbol{\Theta})^{H} \boldsymbol{\Pi}_{\mathrm{c}}^{\perp} \mathbf{x}\right|^{2}}{\left(\mathbf{d}(\boldsymbol{\Theta})^{H} \boldsymbol{\Pi}_{\mathrm{c}}^{\perp} \mathbf{d}(\boldsymbol{\Theta})\right)\left(\mathbf{x}^{H} \boldsymbol{\Pi}_{\mathrm{c}}^{\perp} \mathbf{x}\right)} \underset{H_{0}}{\stackrel{H_{1}}{\gtrless}} \eta
$$

\footnotetext{
${ }^{1}$ The rank is assumed to be known in this paper as in STAP applications according to Brennan's formula [30]: $r=N+$ $(M-1) * \beta$ ( $N$ is the number of sensors, $M$ the number of pulses and $\beta$ depends on radar parameters). If not, it is possible to estimate it for example with new methods based on Random Matrix Theory tools [31].
} 
where $\underset{H_{0}}{\stackrel{H_{1}}{\gtrless}} \eta$ means that the $H_{1}$ hypothesis (respectively $H_{0}$ ) is decided if the test $\Lambda_{\mathrm{LR}}(\Theta)$ is over (respectively under) the threshold $\eta$. In the following, the parameter $\Theta$ is omitted.

Since $\Pi_{\mathrm{c}}^{\perp}$ is not known in practice, we have to estimate it from the secondary data $\left\{\mathbf{x}_{k}\right\}$. The classical estimation is based on the Eigenvalue Decomposition of the Sample Covariance Matrix (SCM):

$$
\hat{\mathbf{R}}=\frac{1}{K} \sum_{k=1}^{K} \mathbf{x}_{k} \mathbf{x}_{k}^{H}=\sum_{i=1}^{r} \hat{\lambda}_{i} \hat{\mathbf{u}}_{i} \hat{\mathbf{u}}_{i}^{H}+\sum_{i=r+1}^{m} \hat{\lambda}_{i} \hat{\mathbf{u}}_{i} \hat{\mathbf{u}}_{i}^{H}
$$

where $\hat{\lambda}_{i}$ and $\hat{\mathbf{u}}_{i}$ are the estimated eigenvalues and eigenvectors. Finally, the estimated projectors are:

$$
\begin{aligned}
\hat{\mathbf{\Pi}}_{\mathrm{c}} & =\sum_{i=1}^{r} \hat{\mathbf{u}}_{i} \hat{\mathbf{u}}_{i}^{H} \\
\hat{\boldsymbol{\Pi}}_{\mathrm{c}}^{\perp} & =\mathbf{I}_{m}-\hat{\boldsymbol{\Pi}}_{\mathrm{c}}=\sum_{i=r+1}^{m} \hat{\mathbf{u}}_{i} \hat{\mathbf{u}}_{i}^{H}
\end{aligned}
$$

Then, using the estimate $\hat{\Pi}_{\mathrm{c}}^{\perp}$, the LR-Adaptive NMF (LR-ANMF) detector can be written as:

$$
\hat{\Lambda}_{\mathrm{LR}}=\frac{\left|\mathbf{d}^{H} \hat{\boldsymbol{\Pi}}_{\mathrm{c}}^{\perp} \mathbf{x}\right|^{2}}{\left(\mathbf{d}^{H} \hat{\boldsymbol{\Pi}}_{\mathrm{c}}^{\perp} \mathbf{d}\right)\left(\mathbf{x}^{H} \hat{\boldsymbol{\Pi}}_{\mathrm{c}}^{\perp} \mathbf{x}\right)} \underset{H_{0}}{\stackrel{H_{1}}{\gtrless}} \eta
$$

\section{ApProximate Distribution OF THE LR-ANMF Under $H_{0}$ HyPOTHESIS}

As in previous works on LR-STAP theoretical performance analysis [13], [14], [26], [27], [28], the following usual assumption is made for mathematical tractability: the projection of the steering vector onto the true interference subspace is negligible, i.e. $\mathbf{u}_{i}^{H} \mathbf{d} \approx 0$ for $i=1, \ldots, r$. This just means that the tested steering vector is not fully embedded in the clutter ridge. We will check in next section by a simulation that the theoretical result is also valid even in a case of non orthogonality of the tested steering vector with respect to the clutter subspace. From the structure of $\mathbf{R}$, we have the following relations:

$$
\mathbf{R d}=\lambda \mathbf{d} \quad, \quad \mathbf{R}^{-1} \mathbf{d}=\frac{1}{\lambda} \mathbf{d} \quad \text { and } \quad \boldsymbol{\Pi}_{c}^{\perp} \mathbf{d}=\mathbf{d}
$$

Without loss of generality, we decide that the norm of steering vector $\mathbf{d}$ is equal to 1 . We finally assume that the CNR is large which leads to: $\lambda_{1}, \ldots, \lambda_{r} \gg \lambda$. This assumption is realistic in many applications as in STAP. Moreover, it is well known that in low CNR regime, a simple detector without the estimated covariance matrix or the estimated projector is good enough. Nevertheless as for the orthogonality assumption, we will perform a simulation in the next section under low CNR conditions to check the validity of our theoretical result.

Main result is given in the next subsection while its proof is next derived in the last subsection. 


\section{A. Main result}

Proposition 3.1: The LR-ANMF of Eq. (10), $\hat{\Lambda}_{\mathrm{LR}}$, can be expressed as a function of 6 independent random variables:

$$
\hat{\Lambda}_{L R} \sim \hat{\Lambda}_{L R_{t h}}=\frac{|\alpha|^{2}}{\beta}
$$

with

$$
\begin{aligned}
\alpha & =b_{1}-\frac{1}{K} X_{1} X_{3} s_{1} \\
\beta & =\left|b_{1}\right|^{2}+X_{2}^{2}-\frac{2}{K} X_{1} X_{3} \Re\left(b_{1} s_{1}+X_{2} s_{2}\right)
\end{aligned}
$$

where $X_{1} \sim \sqrt{\frac{1}{2} \chi^{2}(2 r)}, X_{2} \sim \sqrt{\frac{1}{2} \chi^{2}(2(m-r-1))}, X_{3} \sim \sqrt{\frac{1}{2} \chi^{2}(2 K)}, s_{1} \sim \mathcal{N}_{c}(0,1), s_{2} \sim \mathcal{N}_{c}(0,1)$ and $b_{1} \sim \mathcal{N}_{c}(0,1)$ are independent random variables.

Let us discuss about the meaning of this main result. First, we recall that this result is obtained by a first order approximation. Therefore, it is an approximate distribution of the LR-ANMF under the $H_{0}$ hypothesis. Nevertheless, this result leads to interesting remarks. First by inspecting proposition 3.1, we conclude that the approximate distribution of the LR-ANMF under $H_{0}$ does not depend on the structure of the clutter subspace. Therefore, the LR-ANMF is approximately CFAR: under $H_{0}$, it depends only on the rank, $r$, the data size $m$ and the number of secondary data $K$. To determine a threshold as a function of the Pfa, we need only to perform a Monte Carlo simulation and the time computation is here instantaneous whereas a Monte Carlo simulation of the Eq. (10) could be dramatically important especially for large $m$, involving in that case very large matrices. Moreover, the simulation of the clutter could be difficult in several practical cases (the clutter scenario is not always known in practice). Finally, the hypothesis that the projection of the steering vector on the true interference subspace is negligible is not critical under $H_{0}$ hypothesis. Indeed as in classical adaptive detectors, it will be difficult in practical cases to set a threshold as a function of the parameters contained in $\Theta$ (e.g. in STAP, the angle of arrival and the target speed). Moreover, we will inspect in the simulation section that the derived result in proposition 3.1 remains valid even if this hypothesis is not completely fulfilled (the same study has been also made in [28]).

\section{B. Proof of proposition 3.1}

The proof is first based on the two following propositions:

Proposition 3.2: Define $\Delta \mathbf{R}$ as $\Delta \mathbf{R}=\hat{\mathbf{R}}-\mathbf{R}$. Up to the first order with respect to $\Delta \mathbf{R}$, we have:

$$
\begin{aligned}
\hat{\Lambda}_{\mathrm{LR}} \approx \hat{\Lambda}_{\mathrm{LR}_{1}}= & \frac{\left|\mathbf{d}^{H} \Pi_{c}^{\perp} \mathbf{x}-\mathbf{d}^{H} \boldsymbol{\Pi}_{c}^{\perp} \Delta \mathbf{R} \mathbf{M x}-\mathbf{d}^{H} \mathbf{M} \Delta \mathbf{R} \boldsymbol{\Pi}_{c}^{\perp} \mathbf{x}\right|^{2}}{\left(\mathbf{d}^{H} \Pi_{c}^{\perp} \mathbf{d}-\mathbf{d}^{H} \Pi_{c}^{\perp} \Delta \mathbf{R} \mathbf{M d}-\mathbf{d}^{H} \mathbf{M} \Delta \mathbf{R} \Pi_{c}^{\perp} \mathbf{d}\right)}, \\
& \cdot \frac{1}{\left(\mathbf{x}^{H} \Pi_{c}^{\perp} \mathbf{x}-\mathbf{x}^{H} \Pi_{c}^{\perp} \Delta \mathbf{R} \mathbf{M} \mathbf{x}-\mathbf{x}^{H} \mathbf{M} \Delta \mathbf{R} \Pi_{c}^{\perp} \mathbf{x}\right)}
\end{aligned}
$$


where $\mathbf{M}$ is the pseudo-inverse of $\mathbf{C}$ :

$$
\mathbf{M}=\sum_{i=1}^{r} \frac{1}{\lambda_{i}} \mathbf{u}_{i} \mathbf{u}_{i}^{H}
$$

Proposition 3.3:

$$
\hat{\Lambda}_{\mathrm{LR}_{1}} \approx \hat{\Lambda}_{\mathrm{LR}_{2}}=\frac{|\alpha|^{2}}{\beta}
$$

with

$$
\begin{aligned}
\alpha & =\lambda^{1 / 2}\left(\mathbf{d}^{H} \mathbf{b}-\mathbf{d}^{H} \mathbf{S y}\right) \\
\beta & =\lambda\left(\|\mathbf{b}\|^{2}-2 \Re\left(\mathbf{b}^{H} \mathbf{S y}\right)\right)
\end{aligned}
$$

where

$$
\begin{aligned}
\mathbf{b}, \mathbf{b}_{k} & \sim \mathcal{C N}\left(\mathbf{0}, \boldsymbol{\Pi}_{c}^{\perp}\right) \\
\mathbf{y}, \mathbf{y}_{k} & \sim \mathcal{C N}\left(\mathbf{0}, \boldsymbol{\Pi}_{c}\right) \\
\mathbf{S} & =\frac{1}{K} \sum_{k=1}^{K} \mathbf{b}_{k} \mathbf{y}_{k}^{H}
\end{aligned}
$$

The proofs of both propositions are given in the appendix.

In the sequel, the parameter $\lambda$ is omitted in $\alpha$ and $\beta$ because it cancels in the ratio (16). From Eq. (16), the proof is next based on some invariance properties of $\hat{\Lambda}_{L R_{2}}$ with respect to unitary matrices. Let $\mathbf{Q}_{1}$ and $\mathbf{Q}_{2}$ be two unitary matrices:

$$
\alpha=\mathbf{d}^{H} \mathbf{Q}_{1}^{H} \mathbf{Q}_{1} \mathbf{b}-\mathbf{d}^{H} \mathbf{Q}_{1}^{H} \mathbf{Q}_{1} \mathbf{S} \mathbf{Q}_{2}^{H} \mathbf{Q}_{2} \mathbf{e}
$$

Matrix $\mathbf{Q}_{1}$ is chosen so that $\mathbf{Q}_{1} \mathbf{d}=\mathbf{e}_{1}$ and $\mathbf{Q}_{1} \boldsymbol{\Pi}_{c}^{\perp} \mathbf{Q}_{1}^{H}=\left(\begin{array}{cc}\mathbf{I}_{m-r} & \mathbf{0} \\ \mathbf{0} & \mathbf{0}\end{array}\right)$. Matrix $\mathbf{Q}_{2}$ is chosen so that $\mathbf{Q}_{2} \boldsymbol{\Pi}_{c} \mathbf{Q}_{2}^{H}=\left(\begin{array}{cc}\mathbf{0} & \mathbf{0} \\ \mathbf{0} & \mathbf{I}_{r}\end{array}\right)$. In this way,

$$
\begin{aligned}
\alpha & =\mathbf{e}_{1}^{H} \tilde{\mathbf{b}}-\mathbf{e}_{1}^{H} \tilde{\mathbf{S}} \tilde{\mathbf{y}} \\
\beta & =\|\tilde{\mathbf{b}}\|^{2}-2 \Re\left(\tilde{\mathbf{b}}^{H} \tilde{\mathbf{S}} \tilde{\mathbf{y}}\right)
\end{aligned}
$$

where $\tilde{\mathbf{y}}, \tilde{\mathbf{y}}_{k} \sim \mathcal{N C}\left(\mathbf{0},\left(\begin{array}{cc}\mathbf{0} & \mathbf{0} \\ \mathbf{0} & \mathbf{I}_{r}\end{array}\right)\right), \tilde{\mathbf{b}}, \tilde{\mathbf{b}}_{k} \sim \mathcal{N C}\left(\mathbf{0},\left(\begin{array}{cc}\mathbf{I}_{m-r} & \mathbf{0} \\ \mathbf{0} & \mathbf{0}\end{array}\right)\right)$ and $\tilde{\mathbf{S}}=\frac{1}{K} \sum_{k=1}^{K} \tilde{\mathbf{b}}_{k} \tilde{\mathbf{y}}_{k}^{H}$ 
Simplification with respect to $\tilde{\mathbf{y}}$ : We can write:

$$
\tilde{\mathbf{y}}=X_{1} \mathbf{u} \text { with } X_{1}=\sqrt{\frac{1}{2} \chi^{2}(2 r)}
$$

where $\mathbf{u}$ is a unitary vector and $X_{1}$ is independent of $\mathbf{u}$. It is easy to check using invariance properties with respect to unitary matrices transformation that the distributions of $\alpha$ and $\beta$ conditioned on $\mathbf{u}$ do not depend on $\mathbf{u}$. Then, the distributions of $\alpha$ and $\beta$ are equal to their conditional distributions and we can choose $\mathbf{u}$ at our convenience. We take $\mathbf{u}=\mathbf{e}_{m-r+1}$ which yields:

$$
\begin{aligned}
\alpha & =\mathbf{e}_{1}^{H} \tilde{\mathbf{b}}-X_{1} \mathbf{e}_{1}^{H} \tilde{\mathbf{S}} \mathbf{e}_{m-r+1} \\
\beta & =\|\tilde{\mathbf{b}}\|^{2}-2 X_{1} \Re\left(\tilde{\mathbf{b}}{ }^{H} \tilde{\mathbf{S}} \mathbf{e}_{m-r+1}\right)
\end{aligned}
$$

Simplification with respect to $\tilde{\mathbf{b}}$ : We can write:

$$
\tilde{\mathbf{b}}=b_{1} \mathbf{e}_{1}+X_{2} \mathbf{u} \text { with } X_{2}=\sqrt{\frac{1}{2} \chi^{2}(2(m-r-1))}
$$

where $\mathbf{u}=\left(0 u_{2} \ldots u_{m-r} 0 \ldots 0\right)^{T}$ is an unitary vector composed of $m-r-1$ non-zero values. $X_{2}$ is independent of $\mathbf{u}$ and $b_{1} \sim \mathcal{N}_{c}(0,1)$. As for the previous simplification, we check using invariance properties with respect to unitary matrices transformation that the distributions of $\alpha$ and $\beta$ conditioned on $\mathbf{u}$ do not depend on $\mathbf{u}$. Then, the distributions of $\alpha$ and $\beta$ are equal to their conditional distributions and we can choose $\mathbf{u}$ at our convenience. We take $\mathbf{u}=\mathbf{e}_{2}$ which yields:

$$
\begin{aligned}
\alpha & =b_{1}-X_{1} \mathbf{e}_{1}^{H} \tilde{\mathbf{S}} \mathbf{e}_{m-r+1} \\
\beta & =\left|b_{1}\right|^{2}+X_{2}^{2}-2 X_{1} \Re\left(\left(b_{1} X_{2} 0 \ldots 0\right)^{H} \tilde{\mathbf{S}} \mathbf{e}_{m-r+1}\right)
\end{aligned}
$$

Simplification with respect to $\tilde{\mathbf{S}}$ : Let us study the term $\left(b_{1} X_{2} 0 \ldots 0\right)^{H} \tilde{\mathbf{S}} \mathbf{e}_{m-r+1}$ in Eq. (24):

$$
\begin{aligned}
& \left(b_{1} X_{2} 0 \ldots 0\right)^{H} \tilde{\mathbf{S}} \mathbf{e}_{m-r+1}= \\
& \frac{1}{K}\left(b_{1} X_{2} 0 \ldots 0\right)^{H}\left(\tilde{\mathbf{b}}_{1} \ldots \tilde{\mathbf{b}}_{K}\right)\left(\begin{array}{c}
\tilde{\mathbf{y}}_{1}^{H} \\
\vdots \\
\tilde{\mathbf{y}}_{K}^{H}
\end{array}\right)\left(\begin{array}{c}
0 \\
\vdots \\
1 \\
\vdots \\
0
\end{array}\right) .
\end{aligned}
$$


The result of the multiplication of the two last terms is a vector where each element is $\mathcal{N}_{c}(0,1)$ distributed. Therefore, these two last terms of Eq. (25) can be written as follows:

$$
\left(\begin{array}{c}
\tilde{\mathbf{y}}_{1}^{H} \\
\vdots \\
\tilde{\mathbf{y}}_{K}^{H}
\end{array}\right)\left(\begin{array}{c}
0 \\
\vdots \\
1 \\
\vdots \\
0
\end{array}\right)=X_{3} \mathbf{u} \text { with } X_{3}=\sqrt{\frac{1}{2} \chi^{2}(2 K)}
$$

and $\mathbf{u}$ an independent unitary vector with $K$ components. By using $X_{3}$, Eq. (25) becomes:

$$
\begin{aligned}
& \left(b_{1} X_{2} 0 \ldots 0\right)^{H} \tilde{\mathbf{S e}}_{m-r+1}= \\
& \frac{1}{K} X_{3}\left(b_{1} X_{2} 0 \ldots 0\right)^{H}\left(\tilde{\mathbf{b}}_{1} \ldots \tilde{\mathbf{b}}_{K}\right) \mathbf{u} \\
& =\frac{1}{K} X_{3}\left(b_{1} X_{2} 0 \ldots 0\right)^{H}\left(\begin{array}{cc}
s_{1} & \ldots \\
s_{2} & \ldots \\
\vdots & \vdots \\
\ldots & \ldots
\end{array}\right) \mathbf{u}
\end{aligned}
$$

where only the two first lines are of interest. They are i.i.d with each element is $\mathcal{N}_{c}(0,1)$ distributed. As for the first and the second simplifications, we check using invariance properties with respect to unitary matrices transformation that the distributions of $\alpha$ and $\beta$ conditioned on $\mathbf{u}$ do not depend on $\mathbf{u}$. Then, the distributions of $\alpha$ and $\beta$ are equal to their conditional distributions and we can choose $\mathbf{u}$ at our convenience. We take $\mathbf{u}=\mathbf{e}_{1}$. Then, $\alpha$ and $\beta$ can be rewritten as given in the proposition which concludes the proof.

\section{NumericAl Simulations}

To validate our theoretical result, the STAP processing application is chosen. The purpose of STAP is to detect a moving target thanks to an uniform linear antenna composed of $N$ sensors receiving $M$ pulses. The response of the ground, the clutter $\mathbf{c}$, is the superposition of a large number of points, the clutter patches, allocated at a fixed distance around the airborne radar. According to Brennan's formula [30], the clutter rank is known and is lower than the data size. In STAP application, $\boldsymbol{\Theta}=(\theta ; v)$ where $\theta$ is the DoA (Direction of Arrival) and $v$ the object relative speed.

\section{A. Parameters}

We consider the following STAP configuration. The number $N$ of sensors is 8 and the number $M$ of coherent pulses is also $8(m=N M=64)$. The center frequency and the bandwidth are respectively 
equal to $f_{0}=450 \mathrm{MHz}$ and $B=4 \mathrm{MHz}$. The radar velocity is $100 \mathrm{~m} / \mathrm{s}$. The inter-element spacing is $d=\frac{c}{2 f_{0}}\left(c\right.$ is the celerity of light) and the pulse repetition frequency is $f_{r}=600 \mathrm{~Hz}$. The clutter rank is computed from Brennan rule [30] and is equal to $r=15$. Therefore, the clutter has a low-rank structure since $r=15<m=64$.

The CM C of the Gaussian clutter is computed using the model presented in [29]. The identity matrix is next added to build the CM R. The CNR is defined by:

$$
C N R=\frac{\operatorname{Tr}(C)}{\lambda}
$$

For the steering vector $\mathbf{d}$, we always choose a DoA equal to $0^{\circ}$. The choice of the speed will depend on the simulation. For large speed, the assumption $\mathbf{u}_{i}^{H} \mathbf{d} \approx 0$ for $i \leq r$ will be fulfilled. On the contrary, the hypothesis is no more valid when the speed becomes small.

In the same STAP configuration, $K$ secondary data have been simulated. These secondary data allow us to obtain the SCM $\hat{\mathbf{R}}$. From the EVD of the SCM, the adaptive detector LR-ANMF of Eq. (10), $\hat{\Lambda}_{L R}$, is computed. Moreover, the first order version of the LR-ANMF of Eq. (14), $\hat{\Lambda}_{L R_{1}}$, is also obtained. The final computation of the LR-ANMF of Eq. (16), $\hat{\Lambda}_{L R_{t h}}$, is easily obtained by the trials of only 6 random variables. We also give the LR detector distribution [15] derived with the true projector, denoted LR-NMF and given in Eq. (6).

\section{B. Validation}

First, results for Pfa as function of the threshold are shown in Fig. 1 for $K=m$ and for $K=$ $2 r$. The difference between the LR-ANMF distribution given by the Monte Carlo simulation and our approximate distribution is the same for both values of $K$. We also notice that the LR-ANMF and LRNMF distributions are very close for $K=m$ whereas the LR-ANMF distribution is close to the proposed approximate distribution for $K=2 r$. This result is really interesting when using the LR-ANMF detector in standard conditions for $K$ : for a given Pfa, it allows to determine a threshold more precisely than the LR-NMF distribution. We can also notice that the distributions of $\hat{\Lambda}_{L R_{1}}$ and $\hat{\Lambda}_{L R_{t h}}$ are very close which shows that the error comes mainly from the first order approximation.

For a threshold of $\eta=-10 d B$, we compute the Pfa for the three detectors $\hat{\Lambda}_{L R}, \hat{\Lambda}_{L R_{1}}, \hat{\Lambda}_{L R_{t h}}$ as a function of $K$ and the result is shown in Fig. 2. We notice that the error increases as $K$ decreases. Since the theoretical result is based on a first order approximation, this result is logical.

Now, we propose to validate our theoretical result with respect to the two hypothesis: $\mathbf{u}_{i}^{H} \mathbf{d} \approx 0$ for $\forall i \leq r$ and large CNR. The behavior of the Pfa as a function of the CNR in the top of Fig. 3 is shown. 

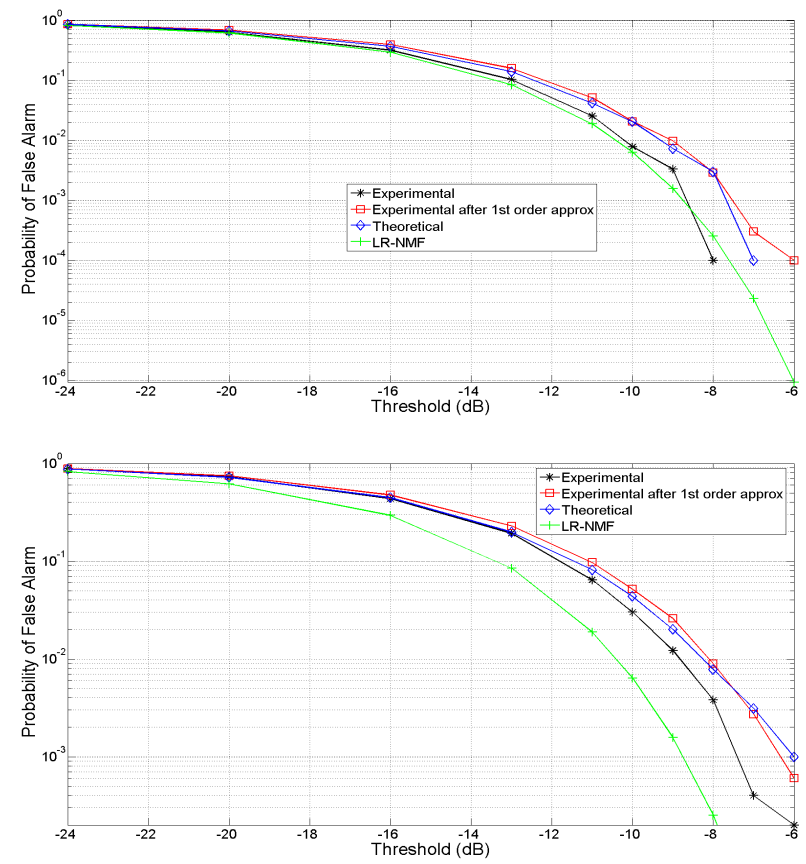

Fig. 1: Pfa as a function of threshold evaluated with 100000 iterations. For $\hat{\Lambda}_{L R}$ (black star), $\hat{\Lambda}_{L R_{1}}$ (square red), $\hat{\Lambda}_{L R_{t h}}$ (blue diamond) and $\Lambda_{L R}$ (green plus). $K=m$ on the top and $K=2 r$ on the bottom. $m=64, r=15, C N R=30 d B$. Large target speed $\left(V_{t}=10 \mathrm{~m} / \mathrm{s}\right)$.

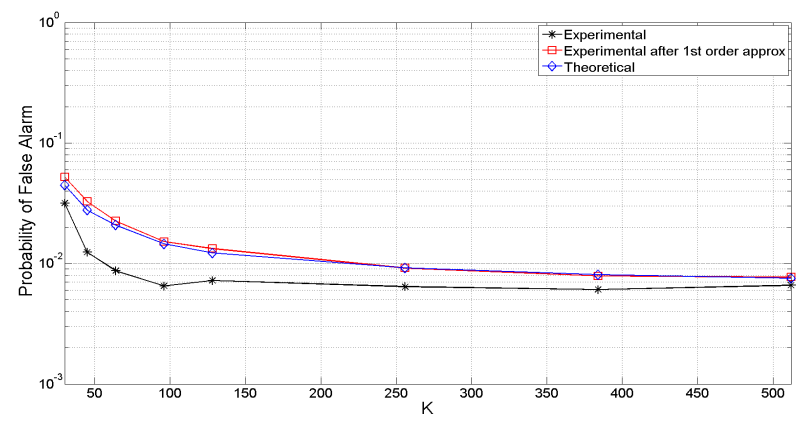

Fig. 2: Pfa as a function of $\mathrm{K}$ evaluated with 100000 iterations. For $\hat{\Lambda}_{L R}$ (black star), $\hat{\Lambda}_{L R_{1}}$ (square red) and $\hat{\Lambda}_{L R_{t h}}$ (blue diamond). $m=64, r=15, C N R=30 d B, \eta=-10 d B$. Large target speed $\left(V_{t}=10 \mathrm{~m} / \mathrm{s}\right)$.

The bottom of Fig. 3 shows the Pfa as a function of the tested speed (parameter of the steering vector d). When the speed is close to $0 \mathrm{~m} / \mathrm{s}$, we recall that the assumption $\mathbf{u}_{i}^{H} \mathbf{d} \approx 0$ is not valid anymore. Both results show that our theoretical result is valid even for small CNR and small tested speed (where the assumptions are no more valid). We conclude that these assumptions needed to derive the theoretical 
calculus are not critical in practical issues.
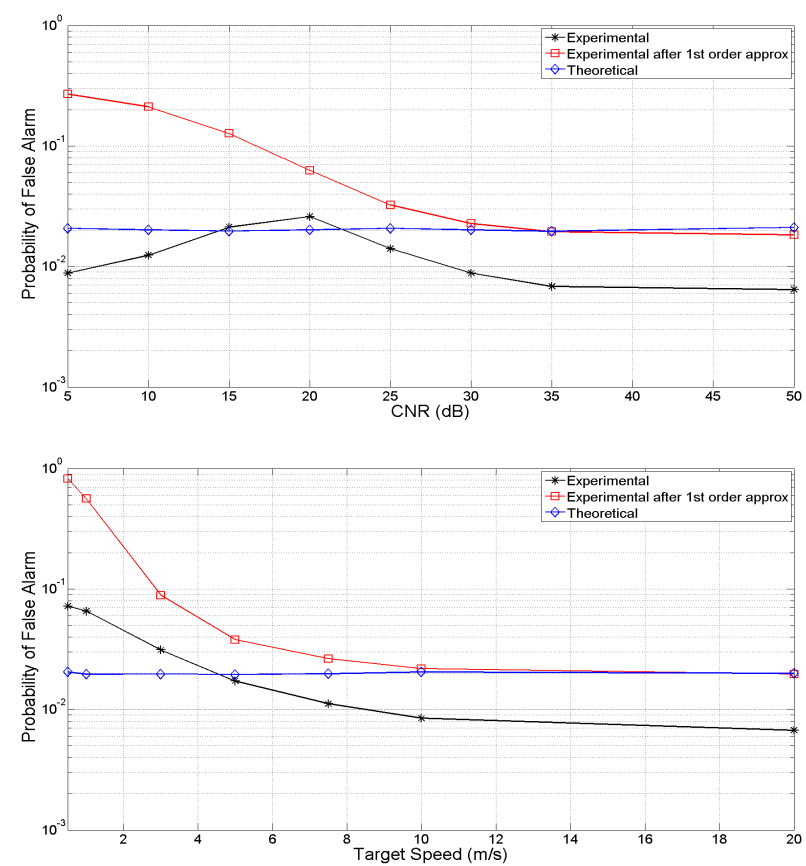

Fig. 3: Pfa as a function of CNR with $V_{t}=10 \mathrm{~m} / \mathrm{s}$ (top) and of the tested speed with $C N R=30 d B$ (bottom) evaluated with 100000 iterations. For $\hat{\Lambda}_{L R}$ (black star), $\hat{\Lambda}_{L R_{1}}$ (square red) and $\hat{\Lambda}_{L R_{t h}}$ (blue diamond). $m=64, r=15, K=m$, $\eta=-10 d B$.

\section{CONCLUSION}

In this paper, we derived an approximate distribution of the LR-ANMF under the null hypothesis. The disturbance is composed of a Gaussian LR clutter plus an AWGN and the estimated projector is built from the EVD of the SCM. The approach has been based on a perturbation analysis. The obtained result showed that the LR-ANMF is approximately CFAR. Moreover, it allows to provide a quasi-instantaneous threshold for a given Pfa without any knowledge of the clutter scenario and more precisely than the theoretical distribution of the LR-NMF. In a STAP context, we validated our theoretical result and showed its good robustness to the different hypotheses.

Further theoretical investigations must be conducted to investigate the limits of our theoretical result in terms of CNR and distance to the clutter subspace. An extension to non Gaussian distributions could be also considered. 


\section{APPENDIX}

\section{A. Proof of proposition 3.2}

Since all considered estimators have been shown consistent, the LR-ANMF is evaluated for large $K$ by means of a perturbation analysis [25]. Starting from the perturbations on $\hat{\mathbf{R}}, \hat{\boldsymbol{\Pi}}_{c}$ and $\hat{\boldsymbol{\Pi}}_{c}^{\perp}$, the estimated LR-ANMF detector of Eq. (10) is reduced to a compact form thanks to a first order approximation.

Let $\Delta \mathbf{R}=\hat{\mathbf{R}}-\mathbf{R}$ be the covariance estimation error on $\mathbf{R}$. This estimation error induces an error on the estimates $\hat{\boldsymbol{\Pi}}_{c}$ and $\hat{\boldsymbol{\Pi}}_{c}^{\perp}$. It is shown in [25] that the projector estimates are given up to the first order with respect to $\Delta \mathbf{R}$ by:

$$
\begin{aligned}
\hat{\boldsymbol{\Pi}}_{c} & \approx \boldsymbol{\Pi}_{c}+\delta \boldsymbol{\Pi}_{c} \\
\hat{\boldsymbol{\Pi}}_{c}^{\perp} & \approx \boldsymbol{\Pi}_{c}^{\perp}-\delta \boldsymbol{\Pi}_{c}
\end{aligned}
$$

where $\delta \boldsymbol{\Pi}_{c}$ is equal to:

$$
\delta \boldsymbol{\Pi}_{c}=\boldsymbol{\Pi}_{c}^{\perp} \Delta \mathbf{R M}+\mathbf{M} \Delta \mathbf{R} \Pi_{c}^{\perp}
$$

In what follows, all equalities are valid up to the first order with respect to $\Delta \mathbf{R}$.

By replacing Eqs. (29) and (30) in Eq. (10), we finish the proof.

\section{B. Proof of proposition 3.3}

The numerator of Eq. (14) can be expressed as:

$$
\begin{aligned}
& \mathbf{d}^{H} \Pi_{c}^{\perp} \mathbf{x}-\mathbf{d}^{H} \Pi_{c}^{\perp} \Delta \mathbf{R M x}-\mathbf{d}^{H} \mathbf{M} \Delta \mathbf{R} \Pi_{c}^{\perp} \mathbf{x}= \\
& \mathbf{d}^{H} \Pi_{c}^{\perp} \mathbf{x}-\mathbf{d}^{H} \Pi_{c}^{\perp} \Delta \mathbf{R M x}=\mathbf{d}^{H} \Pi_{c}^{\perp} \mathbf{x}-\mathbf{d}^{H} \Pi_{c}^{\perp} \hat{\mathbf{R}} \mathbf{M x}
\end{aligned}
$$

since $\mathbf{d}^{H} \mathbf{M}=\mathbf{0}$ and $\Delta \mathbf{R}$ can be replaced by $\hat{\mathbf{R}}$ because $\mathbf{d}^{H} \boldsymbol{\Pi}_{c}^{\perp} \mathbf{R M x}=0$. Let us introduce the following variables:

$$
\begin{aligned}
& \mathbf{\Pi}_{c}^{\perp} \mathbf{x}=\left(\boldsymbol{\Pi}_{c}^{\perp} \mathbf{n}\right)=\lambda^{1 / 2} \mathbf{b} \text { where } \mathbf{b} \sim \mathcal{C N}\left(\mathbf{0}, \boldsymbol{\Pi}_{c}^{\perp}\right) \\
& \mathbf{M}^{1 / 2} \mathbf{x}=\mathbf{M}^{1 / 2} \mathbf{c}+\mathbf{M}^{1 / 2} \mathbf{n} \approx \mathbf{M}^{1 / 2} \mathbf{c}=\mathbf{y} \sim \mathcal{C N}\left(\mathbf{0}, \boldsymbol{\Pi}_{c}\right)
\end{aligned}
$$

For the second variable, the strong CNR hypothesis has been used. Similarly:

$$
\begin{aligned}
& \boldsymbol{\Pi}_{c}^{\perp} \hat{\mathbf{R}} \mathbf{M}^{1 / 2}=\boldsymbol{\Pi}_{c}^{\perp} \frac{1}{K} \sum_{k=1}^{K} \mathbf{x}_{k} \mathbf{x}_{k}^{H} \mathbf{M}^{1 / 2} \\
& =\frac{\lambda^{1 / 2}}{K} \sum_{k=1}^{K} \mathbf{b}_{k} \mathbf{y}_{k}^{H}=\lambda^{1 / 2} \mathbf{S}
\end{aligned}
$$

and Eq. (31) becomes finally:

$$
\mathbf{d}^{H} \hat{\mathbf{\Pi}}_{c}^{\perp} \mathbf{x}=\lambda^{1 / 2}\left(\mathbf{d}^{H} \mathbf{b}-\mathbf{d}^{H} \mathbf{S y}\right)
$$

Let us turn to the denominator. From hypotheses on d, we have for the first term of Eq. (14):

$$
\mathbf{d}^{H} \boldsymbol{\Pi}_{c}^{\perp} \mathbf{d}-\mathbf{d}^{H} \boldsymbol{\Pi}_{c}^{\perp} \Delta \mathbf{R} \mathbf{M d}-\mathbf{d}^{H} \mathbf{M} \Delta \mathbf{R} \boldsymbol{\Pi}_{c}^{\perp} \mathbf{d}=1
$$


For the second term, we have similarly:

$$
\begin{aligned}
& \mathbf{x}^{H} \boldsymbol{\Pi}_{c}^{\perp} \mathbf{x}-\mathbf{x}^{H} \boldsymbol{\Pi}_{c}^{\perp} \Delta \mathbf{R M} \mathbf{x}-\mathbf{x}^{H} \mathbf{M} \Delta \mathbf{R} \boldsymbol{\Pi}_{c}^{\perp} \mathbf{x}= \\
& \mathbf{x}^{H} \boldsymbol{\Pi}_{c}^{\perp} \mathbf{x}-\mathbf{x}^{H} \boldsymbol{\Pi}_{c}^{\perp} \hat{\mathbf{R}} \mathbf{M} \mathbf{x}-\mathbf{x}^{H} \mathbf{M} \hat{\mathbf{R}} \boldsymbol{\Pi}_{c}^{\perp} \mathbf{x}= \\
& \lambda \mathbf{b}^{H} \mathbf{b}-\lambda^{1 / 2} \mathbf{b}^{H} \boldsymbol{\Pi}_{c}^{\perp} \hat{\mathbf{R}} \mathbf{M}^{1 / 2} \mathbf{y}-\lambda^{1 / 2} \mathbf{y}^{H} \mathbf{M}^{1 / 2} \hat{\mathbf{R}} \boldsymbol{\Pi}_{c}^{\perp} \mathbf{b}
\end{aligned}
$$

By using the matrix $\mathbf{S}$ of Eq. (18), we have:

$$
\begin{aligned}
& \mathbf{x}^{H} \boldsymbol{\Pi}_{c}^{\perp} \mathbf{x}-\mathbf{x}^{H} \boldsymbol{\Pi}_{c}^{\perp} \Delta \mathbf{R M} \mathbf{x}-\mathbf{x}^{H} \mathbf{M} \Delta \mathbf{R} \boldsymbol{\Pi}_{c}^{\perp} \mathbf{x}= \\
& \lambda\left(\|\mathbf{b}\|^{2}-\mathbf{b}^{H} \mathbf{S y}-\mathbf{y}^{H} \mathbf{S}^{H} \mathbf{b}\right)=\lambda\left(\|\mathbf{b}\|^{2}-2 \Re\left(\mathbf{b}^{H} \mathbf{S y}\right)\right)
\end{aligned}
$$

which concludes the proof.

\section{REFERENCES}

[1] H.L. Van Trees, Estimation and modulation theory, vol. 1, John Wiley and Sons, 2001.

[2] L.L. Scharf and B. Friedlander, "Matched subspace detectors," IEEE Trans. Signal Process., vol. 42, no. 8, pp. 2146-2157, august 1994.

[3] E.J. Kelly, "An adaptive detection algorithm," IEEE Trans. on Aero. and Elec. Syst., vol. 22, no. 1, pp. 115-127, march 1986.

[4] F. Robey, D. Fuhrmann, E. Kelly, and R. Nitzberg, "A CFAR adaptive matched filter detector," IEEE Trans. on Aero. and Elec. Syst., vol. 28, no. 2, pp. 208 - 216, 1992.

[5] I.S. Reed, J.D. Mallett, and L.E. Brennan, "Rapid convergence rate in adaptive arrays," IEEE Trans. on Aero. and Elec. Syst., vol. AES-10, no. 6, pp. 853 - 863, November 1974.

[6] L. B. Fertig, "Analytical expressions for space-time adaptive processing (STAP) performance," IEEE Trans. on Aero.and Elect. Syst., vol. 51, no. 1, pp. 442 - 453, January 2015.

[7] P. Wang, Z. Wang, H. Li, and B. Himed, "Knowledge-aided parametric adaptive matched filter with automatic combining for covariance estimation,” IEEE Trans. Signal Process., vol. 62, no. 18, pp. 4713 - 4722, September 2014.

[8] Y. Tong, T. Wang, and J. Wu, "Improving EFA-STAP performance using persymmetric covariance matrix estimation," IEEE Trans. on Aero. and Elec. Syst., vol. 51, no. 2, pp. 924 - 936, April 2015.

[9] X. Li, D. Feng, H-W. Liu, and D. Luo, "Dimension-reduced space-time adaptive clutter suppression algorithm based on lower-rank approximation to weight matrix in airborne radar," IEEE Trans. on Aero. and Elec. Syst., vol. 50, no. 1, pp. 53 - 69, January 2014.

[10] J. H. Bang, W. L. Melvin, and A. L. Lanterman, "Model-based clutter cancellation based on enhanced knowledge-aided parametric covariance estimation,” IEEE Trans. on Aero.and Elect. Syst., vol. 51, no. 1, pp. 154 - 166, January 2015.

[11] S. Bidon, O. Besson, and J.-Y. Tourneret, "A bayesian approach to adaptive detection in non-homogeneous environments," IEEE Trans. Signal Process., vol. 56, no. 1, pp. 205 - 217, January 2008.

[12] X. Wang, E. Aboutanios, and M. G. Amin, "Reduced-rank STAP for slow-moving target detection by antenna-pulse selection,” IEEE Signal Processing Letters, vol. 22, no. 8, pp. 1156 - 1160, August 2015.

[13] I. Kirsteins and D. Tufts, "Adaptive detection using a low rank approximation to a data matrix," IEEE Trans. on Aero. and Elec. Syst., vol. 30, pp. 55 - 67, 1994.

[14] A. Haimovich, "Asymptotic distribution of the conditional signal-to-noise ratio in an eigenanalysis-based adaptive array," IEEE Trans. on Aero. and Elec. Syst., vol. 33, pp. 988 - 997, 1997. 
[15] M. Rangaswamy, F.C. Lin, and K.R. Gerlach, "Robust adaptive signal processing methods for heterogeneous radar clutter scenarios," Signal Processing, vol. 84, pp. 1653 - 1665, 2004.

[16] M. Rangaswamy, I.P. Kirsteins, B.E. Freburger, and D.W. Tufts, "Low rank adaptive signal processing for radar applications," in Proceedings of ICASSP, Montreal, QC, Canada, 2004, pp. 201-204.

[17] R.S. Raghavan, "Statistical interpretation of a data adaptive clutter subspace estimation algorithm," IEEE Trans. on Aero. and Elec. Syst., vol. 48, no. 2, pp. 1370 - 1384, April 2012.

[18] I. Soloveychik and A. Wiesel, “Tyler's covariance matrix estimator in elliptical models with convex structure," IEEE Trans. Signal Process., vol. 62, no. 20, pp. 5251 - 5259, 2014.

[19] M. B. Kang; Monga, V.; Rangaswamy, "Rank-constrained maximum likelihood estimation of structured covariance matrices,” IEEE Trans. on Aero. and Elec. Syst., vol. 50, no. 1, pp. 501 - 515, 2014.

[20] M. B. Kang; Monga, V.; Rangaswamy, "Computationally efficient toeplitz approximation of structured covariance under a rank constraint,” IEEE Trans. on Aero. and Elec. Syst., vol. 51, no. 1, pp. 775 - 785, january 2015.

[21] A. Breloy, G. Ginolhac, F. Pascal, and P. Forster, "Clutter subspace estimation in low rank heterogeneous noise context," IEEE Trans. Signal Process., vol. 63, no. 9, pp. 2173 - 2182, 2015.

[22] A. Dogandzic and B. Zhang, "Complex signal amplitude estimation and adaptive detection in unknown low-rank interference," in Proceedings of Asilomar Conference, Pacific Grove, CA, USA, November 2006.

[23] A. Dogandzic and B. Zhang, "Bayesian complex amplitude estimation and adaptive matched filter detection in low-rank interference," IEEE Trans. Signal Process., vol. 55, no. 3, pp. 1176 - 1182, march 2007.

[24] J.-F. Degurse, L. Savy, and S. Marcos, "Reduced-rank stap for target detection in heterogeneous environments," IEEE Trans. on Aero. and Elec. Syst., vol. 50, no. 2, pp. 1153 - 1162, april 2014.

[25] H. Krim, P. Forster, and J.G. Proakis, "Operator approach to performance analysis of root-MUSIC and root-min-norm," IEEE Trans. Signal Process., vol. 40, no. 7, pp. 1687 - 1696, July 1992.

[26] C.D. Peckham, A.M. Haimovich, T.F. Ayoub, J.S. Goldstein, and I.S. Reed, "Reduced-rank STAP performance analysis," IEEE Trans. on Aero. and Elec. Syst., vol. 36, no. 2, pp. 664 - 676, April 2000.

[27] G. Ginolhac, P. Forster, F. Pascal, and J.P. Ovarlez, "Performance of two low-rank STAP filters in a heterogeneous noise," IEEE Trans. Signal Process., vol. 61, no. 1, pp. 57 - 61, January 2013.

[28] G. Ginolhac, P. Forster, F. Pascal, and J.P. Ovarlez, "Exploiting persymmetry for low-rank space time adaptive processing," Signal Processing, vol. 97, no. 4, pp. 242 - 251, April 2014.

[29] J. Ward, “Space-Time Adaptive Processing for airborne radar,” Tech. Rep., Lincoln Lab., MIT, Lexington, Mass., USA, December 1994.

[30] L. E. Brennan and F.M. Staudaher, "Subclutter visibility demonstration," Tech. Rep., RL-TR-92-21, Adaptive Sensors Incorporated, 1992.

[31] R. Nadakuditi and J. Silverstein, "Fundamental limit of sample generalized eigenvalue based detection of signals in noise using relatively few signal-bearing and noise-only samples," IEEE Journal of Selected Topics in Signal Processing, vol. 4, no. 3, pp. $468-480,2010$. 


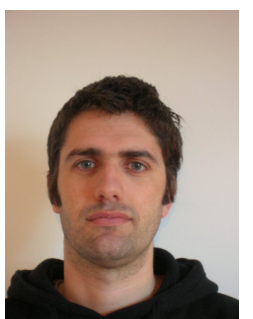

Guillaume Ginolhac was born in France in 1974. He received the degree of electrical engineer in 1997 and a Ph.D. degree in 2001 (signal processing) from the Grenoble-INP University. From 2002 to 2012, he was associate professor of electrical engineering at the Institut Universitaire de Technologie de Ville d'Avray, University Paris X. During this period, he was also a researcher of the Laboratory of Systems and Applications of Information and Energy Technologies (SATIE). He is currently a professor of electrical engineering at the University Savoie Mont-Blanc, France, and a member of the Lab LISTIC. His research interests are in estimation, and detection theory with applications to array processing, radar, and teledetection.

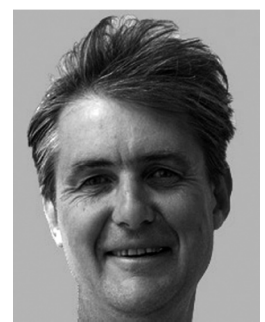

Philippe Forster (M'89) was born in Brest, France, in 1960. He graduated from the Ecole Normale Supérieure de Cachan in 1983 with an Agrégation de Physique Appliquée degree and from the Université de Rennes in 1988 with a Ph.D. degree in electrical engineering. From 1989 to 1992, he worked at Thomson Sintra Activités Sous-Marines, doing research in signal processing for sonar applications. During that time, he spent 1 y at Northeastern University, Boston, as a postdoctoral researcher. In September 1992, he joined the Conservatoire National des Arts et Métiers, Paris, as an assistant professor in electrical engineering, doing research in array processing for mobile communications. Since 2000, he has been a professor of electrical engineering at the Université de Paris X and a member of the SATIE Laboratory at the Ecole Normale Supérieure de Cachan. His research interests are in array processing, detection, and estimation theory. 
Caption of Fig. 1: Pfa as a function of threshold evaluated with 100000 iterations. For $\hat{\Lambda}_{L R}$ (black star), $\hat{\Lambda}_{L R_{1}}$ (square red), $\hat{\Lambda}_{L R_{t h}}$ (blue diamond) and $\Lambda_{L R}$ (green plus). $K=m$ on the top and $K=2 r$ on the bottom. $m=64, r=15$, $C N R=30 d B$. Large target speed $\left(V_{t}=10 \mathrm{~m} / \mathrm{s}\right)$.

Caption of Fig. 2: Pfa as a function of K evaluated with 100000 iterations. For $\hat{\Lambda}_{L R}$ (black star), $\hat{\Lambda}_{L R_{1}}$ (square red) and $\hat{\Lambda}_{L R_{t h}}$ (blue diamond). $m=64, r=15, C N R=30 \mathrm{~dB}, \eta=-10 \mathrm{~dB}$. Large target speed $\left(V_{t}=10 \mathrm{~m} / \mathrm{s}\right)$.

Caption of Fig. 3: Pfa as a function of CNR with $V_{t}=10 \mathrm{~m} / \mathrm{s}$ (top) and of the tested speed with $C N R=30 \mathrm{~dB}$ (bottom) evaluated with 100000 iterations. For $\hat{\Lambda}_{L R}$ (black star), $\hat{\Lambda}_{L R_{1}}$ (square red) and $\hat{\Lambda}_{L R_{t h}}$ (blue diamond). $m=64$, $r=15, K=m, \eta=-10 d B$. 